\title{
POURQUOI REVIENT-ON À SES MOUTONS ? UN REGARD CROISÉ SUR LA VALEUR SÉMANTIQUE DU RETOUR EN FRANÇAIS ET EN POLONAIS
}

\begin{abstract}
A bstrac1. Sikora-Pouivet Dorota, Pourquoi revient-on à ses moutons? Un regard croisé sur la valeur sémantique du retour en français et en polonais [Why "revient-on à ses moutons"? A cross look at the semantic value of the concept of "retour" in French and in Polish]. Studia Romanica Posnaniensia, Adam Mickiewicz University Press, Poznań, vol. XXXIII : 2006, pp. 199-218. ISBN 83-232-1643-6, ISSN 0137-2475.

French verbs revenir, rentrer, retourner refer to the same kind of location situations as their Polish «doubles " wrócić/wracać. This paper analyzes their meanings through examples presented with their contextual data. Revenir and rentrer are morphologically analysable as complex lexemes derived from the corresponding venir and entrer by adding the prefix $R E$-. $R E$ - derivation provides a semantic feature of iterativeness to these complex items. From a synchronic point of view, retourner, in the sensc investigated in this article, cannot be considered anymorc as $R E$ - derived, but it lexicalizes similar values. Wracać/wrócic share some of them. Some differences in the lexical meaning of all these verbs are explained, especially by showing how they are used as pragmatic indexes inside the relation between the speaker and a reference to a location.
\end{abstract}

Pourquoi revient-on à ses moutons plutôt que de retourner les voir? La question semble frivole, mais l'intérêt d'une réponse, tant linguistique que pédagogique, est certain. Quantité d'étudiants en Français Langue Étrangère trébuchent depuis toujours sur les emplois de revenir, rentrer et retourner. Ces verbes sont ressentis, plutôt que compris, comme des synonymes ou, du moins, comme des équivalents discursifs. Dans cet article, nous nous proposons d'observer le fonctionnement de ces items dans différentes situations de communication afin de le comparer ensuite à celui du couple polonais wrócić/wracać.

Dans un premier temps, nous allons nous arrêter sur les trois verbes français mis parfois dans le même panier pour cause de ressemblance. Une réflexion sur leurs propriétés morphologiques, largement inspirée par l'article de Denis Apothéloz sur les différentes manifestations d'itérativité liées au préfixe $R E$ - (voir Apothéloz 2005), devrait permettre de saisir d'une part en quoi consiste le faux air de famille entre ces items lexicaux, et d'autre part de repérer ce qui les distingue. Le 
préfixe $R E$ - y est pour beaucoup, à tout le moins en ce qui conceme revenir et rentrer. Considéré comme foncteur appliqué à la base de dérivation ou à certains de ses éléments, il détermine en grande partie des effets de sens produits par le mot construit. Nous entendons montrer par la suite qu'en choisissant, dans une situation de discours, le mot «juste », adéquat à ce que nous voulons dire, il convient de prendre en compte non seulement les paramètres du référent, mais également de se situer soi-même, en tant que locuteur, par rapport au déroulement du procès. Il s'agirait d'un phénomène déictique. Une troisième partie sera consacrée au couple polonais wracać/wrócic $\hat{c}^{1}$, dont la sémantique sera comparée à celle de ses « cousins » français. Dans notre démarche, nous adoptons résolument une perspective référentielle. Les exemples sont accompagnés d'une description aussi fidèle que possible de la situation d'énonciation. C'est le seul moyen, nous semble-t-il, de ne pas passer à côté des paramètres contextuels ${ }^{2}$, essentiels pour le maniement des verbes qui nous occupent ici.

\section{UN FAUX AIR DE FAMILLE ET QUELQUES FAITS MORPHOLOGIQUES}

1.1. Certains énoncés tels que

(1) Je rentre à la maison.

(2) Je retourne à la maison.

(3) Je reviens à la maison.

pris en dehors de toute situation d'énonciation, peuvent apparaître comme équivalents. De là, il n'y a plus qu'un pas pour conclure à la synonymie de rentrer, revenir et retourner. Il est vrai que, dans ce cas précis, ils lexicalisent les mêmes acceptions que les verbes polonais wracać/wrócić. Cependant, quand il s'agit de rendre compte d'un événement, nous ne pouvons nullement nous fier à cette prétendue synonymie. Elle se trouve rapidement battue en brèche car la situation extra-linguistique dont nous voulons parler force notre choix lexical. Il n'est alors pas indifférent d'employer l'un ou l'autre. Imaginons un parent polonais, en période de vacances, profitant de la plage en compagnie de son enfant. Cédant à ses demandes répétées, il lui donne une pièce et l'autorise à aller s'acheter une glace. Il lui dit alors :

(4) Idż, kup sobie loda i wracaj.

Une substitution pure et simple conduit à des résultats pour le moins curieux. On obtient ainsi :

\footnotetext{
${ }^{1}$ Faute de place, nous ne saurions nous lancer ici dans une analyse de différences aspectuelles et temporelles des doublets polonais.

${ }^{2}$ Nous parlerons ici de contexte au sens large du terme, qui ne se limite pas à l'environnement linguistique de l'énoncé, mais comprend toutes les informations (linguistiques, pragmatiques, socio-culturelles) à la disposition du locuteur pour interpréter le discours (Voir Moeschler J. \& Reboul A., 1994).
} 
(5) Va t'acheter une glace et reviens.

? (6) Va t'acheter une glace et rentre.

? (7) Va t'acheter une glace et retourne (ici).

(6) et (7) paraissent décidément inadéquats à la situation même si c'est pour des raisons différentes. Nous les analyserons dans la section 2 .

1.2. En français, revenir, rentrer et retourner présentent une structure morphologique similaire. Tous les trois relèvent du même paradigme dérivationnel par préfixation en $R E-{ }^{3}$. Dans la mesure où il s'agit d'une classe dérivationnelle des plus productives, on aboutit à une multitude d'items lexicaux en $R E$ - aux significations multiples et variées. On aurait tendance à voir dans $R E$ - un morphème polysémique. En effet, peut-on soutenir le contraire lorsqu'on songe à refaire, à ranimer ou encore à rappeler?

Apothéloz (2005) défend justement la thèse opposée en proposant de réduire cette polysémie souvent encombrante. L'auteur examine le préfixe $R E$ - en tant que foncteur opérant sur un argument $X$. Cet argument n'est autre que la base de dérivation dans la totalité de sa structure actancielle ou bien l'un de ses éléments. $R E$ n'est pas polysémique, bien au contraire. Appliqué à son argument $X$, il apporte à sa base de dérivation l'élément «à nouveau » en produisant un sens itératif. Celui-ci peut ensuite, selon le cas donner lieu soit à une interprétation itérative, soit à une interprétation annulative. Il y aurait ainsi un sens propre au préfixe $R E_{-}$, avec deux effets de sens possibles.

1.2.1. Si elle se laisse résumer par une seule formule «à nouveau $X$ », la valeur itérative de $R E$ - peut s'interpréter de différentes manières selon ce qui tombe sous la portée du foncteur. En suivant le raisonnement et les analyses de l'auteur, nous allons à présent regarder de plus près ces différents cas de figure. Soit le verbe reprendre et les énoncés suivants :

(8) Je reprends de ton rôti, il est excellent.

(9) 11 ne comprend rien à ce que je dis. Reprends tout dès le début, moi, j'abandonne !

(10) Le rétablissement a été très rapide, il reprend le mois prochain.

L'itérativité véhiculée par $R E-{ }^{4}$ peut s'interpréter de trois façons différentes selon la portée du foncteur. En paraphrasant (8), on dira : «à nouveau A prends $x$ ». Le foncteur opère ici sur tous les éléments de la structure actancielle, l'agent comme le patient, ce qui donne lieu à l'itération du procès. (9) présente un deuxième type

\footnotetext{
${ }^{3}$ Nous suivons la convention adoptée dans A pothéloz (2005) : le morphème-type $R E$ - est transcrit en petites capitales, alors que ses différentes réalisations $r e-, r e ́$ - et $r$-scrons notées en miniscules.

${ }^{4}$ Comme le remarque l'auteur, en interprétant les dérivés en $R E$-, il faut bien se garder de confondre la valeur sémantique du foncteur et les multiples facettes de son argument, c'est-à-dire une polysémic éventuelle de la basc de dérivation. Si le reprendre de (8) a un sens différent de celui de (9) ou (10), ce n'est pas à cause de $R E$-, mais justement à cause de prendre.
} 
d'itérativité. Dans cet énoncé, le foncteur porte sur un seul paramètre $x$ (en l'occurrence tout), élément de la structure actancielle de l'argument $X$-prendre. Cela signifie que «à nouveau il y aura $x$ », en sachant que $x=$ une/des explication(s). Ce qui se reproduira, ce n'est pas le procès intégral, mais un événement qui en fait partie. L'agent de (10) reste inchangé, c'est lui qui reprendra, i.e. répètera quelque chose, une activité quelle qu'elle soit. Nous sommes done face à une itération d'activité qui est la troisième interprétation possible d'itérativité présentée par reprendre. Lorsque nous avons affaire à un dérivé en $R E$ - dans son acception itérative, nous présupposons qu'une action similaire ou au moins l'un de ses paramètres, exprimés par le verbe-base de dérivation a déjà eu lieu. " $A$ reprend $x$ » signifie qu'auparavant « $A$ a déjà pris $x$ » : j'avais déjà pris du rôti, quelqu'un a déjà «pris » tous les arguments, il avait déjà « pris » une activité au moins une fois dans sa vie.

1.2.2. Il en est autrement lorsque reprendre (ou un autre dérivé en $R E$-) véhicule une valeur annulative, comme c'est le cas de (11) et (12)

(11) «Rendez-moi, dit-il, mes chansons et mon somme. Et reprenez vos cent écus » (La Fontaine).

(12) Le Royaume Uni a repris la Présidence du Conseil de l'Union européenne.

Dans ce type d'emplois, reprendre signifie «prendre pour entrer à nouveau en possession de quelque chose qu'on avait donné ». Dans son acception annulative, reprendre est en relation d'antonymie directionnelle avec donner (11) ou laisser, abandonner (12), dont il annule les états résultatifs : le foncteur $R E$ - porte sur l'objet censé changer d'emplacement et d'appartenance. Pour qu'il y ait valeur annulative, on doit donc présupposer l'accomplissement préalable de ce que Denis Apothéloz appelle un «procès transformateur (...) directionnellement inverse de celui dénoté par le verbe base $"^{5}$.

1.2.3. Arrêtons-nous un instant sur les critères qui permettent de distinguer les dérivés en $R E$ - des formes qui, malgré une ressemblance de surface, n'en sont pas (ou plus). Il s'agit de faire la différence entre les items morphologiquement construits (comme reprendre, redescendre, remonter, redire) et ceux que, dans une perspective synchronique actuelle, nous devons traiter comme entièrement lexicalisés (par exemple remplacer, rassasier, rallier, rencontrer). On ne peut plus calculer leur sens à partir des apports du préfixe et de las base. A la différence des premiers, ces derniers ont perdu leur compositionnalité. Leur sens effectif s'étant éloigné de celui que l'on pourrait déduire de leur structure, ils sont mémorisés comme lexèmes sémantiquement autonomes.

Les lexèmes construits par préfixation $R E$-, au contraire, gardent leur diagrammaticité et, par conséquent, leur sens est plus ou moins prédictible à partir des éléments formels qui les composent. Rappelons, avec Apothéloz (2005), les trois con-

\footnotetext{
${ }^{5}$ A pothéloz, 2005, p. 61
} 
ditions que doit remplir un item lexical en $R E$ - pour qu'on puisse le considérer comme construit :

(i) il doit contenir dans son sémantisme, à quelque niveau d'analyse que ce soit, l'élément «à nouveau» ;

(ii) cet élément "à nouveau », lié à l'apport sémantique du préfixe foncteur $R E$-, doit porter sur un argument relevant de la structure sémantique de la base de dérivation ;

(iii) le dérivé doit être hyponyme de sa base de dérivation.

\section{REVENIR, RENTRER, RETOURNER EN TANT QUE LEXEMES CONSTRUITS (OU NON ?) EN RE-}

Dans cette section, nous nous proposons de regarder de plus près ces trois verbes. Nous allons vérifier s'il correspondent aux critères établis par Apothéloz et, si tel est le cas, quelle est, pour chacun d'entre eux, la portée du foncteur $R E$-. Ces renseignements devraient jeter une lumière sur l'éventuelle valeur itérative et/ou annulative de ces items.

\subsection{Le cas de revenir}

2.1.1. Y a-t-il un composant «à nouveau » dans la structure sémantique de revenir? La question est, on l'a compris, de savoir si l'item satisfait à la condition (i) évoquée ci-dessus. Soit les exemples (13) et (14):

(13) Toni Braxton revient bientôt.

La diva soul américaine sera bientôt de retour avec un nouveau (sic !) opus et un nouveau single. (presse)

(14) La Coccinelle revient ! (affiche de cinéma)

(13) comme (14) se laissent réduire à une forme minimale " $A$ revient ", intuitivement paraphrasable par " $A$ vient à nouveau ». La condition (i) est ainsi satisfaite. Dans l'univers du discours, les deux énoncés présupposent valide l'information " $A$ est déjà venu auparavant » et appellent de ce fait une interprétation itérative. Nous reprendrons dans la section suivante la réflexion sur la nature de cette itérativité (s'agit-il de l'itération du procès, de l'événement ou d'activité ?) en essayant de délimiter la portée du foncteur RE- dans chacun de ces exemples. Il convient d'abord de reprendre celui que nous avons déjà évoqué dans la section 1. Peut-on trouver ce même composant «à nouveau » dans (5)?

(5) Va t'acheter une glace et reviens !

Pour éviter les difficultés liées à la forme injonctive de (5), que nous n'avons pas la possibilité d'examiner ici, appelons un autre exemple où revenir s'interprète de manière similaire et dont la forme assertive rend la demonstration plus claire : 
(15) Je suis revenu chez Wanadoo. Je viens de quitter Neuf Télécom, son deux mégas et son téléphone illimité à $24,90 €$ pour revenir à mon bon vieux 512 extense sans bonus au même tarif (écrit électronique).

Dans ces emplois, (5) - abstraction faite de sa forme injonctive - comme (15) peuvent être ramenés à l'énoncé minimal « $A$ revient » mais celui-ci appelle une interprétation différente. Nous pouvons la formuler de façon suivante: « $A$ vient pour être à nouveau dans le lieu qu'il a auparavant quitté ». On y retrouve l'élément «à nouveau » imposé par la condition (i). L'information présupposée valide dans l'univers du discours est donc : « $A$ était parti » ou bien « $A$ avait quitté un lieu $P$ », ce qui permet de conclure à un effet de sens annulatif. Partir, quitter ou encore $s$ 'en aller, antonymes lexicalisés de revenir dans les exemples (5) et (15), dénotent le procès transformateur dont nous avons parlé plus haut.

2.1.2. Conformément à la condition (ii), le foncteur $R E$ - avec sa valeur « $\grave{a}$ nouveau $x$ », dont nous avons constaté la présence dans revenir, doit opérer sur un argument relevant de la structure sémantique de la base de dérivation. $R E$ - porte soit sur la structure dans sa totalité (l'exemple 8), soit sur l'un des paramètres qui la compose (9). Si nous voulons comprendre les différentes interprétations possibles de revenir, il nous faut repérer les paramètres liés à la valence de venir susceptibles de tomber dans le champ d'action du foncteur.

2.1.2.1. Les verbes transitifs expriment une relation qui s'établit entre l'agent et le patient ou l'objet du procès qu'ils dénotent. Les verbes de déplacement mettent en rapport un agent (ou dans d'autres cas, une entité) et un point de référence spatiale $P$. Le premier de ces arguments, que Langacker $(1990)^{6}$ appelle « mover » a un statut particulier : il peut être en même temps agent et patient du déplacement. Décrire un déplacement suppose de prendre également en compte le facteur temporel $(T)$. Fillmore $(1975)^{7}$ explique ainsi le rapport entre tous ces paramètres : "Nous disons que quelque chose est en mouvement - par mouvement - j'entends un déplacement - lorsque cet objet est localisé dans un endroit à un moment donné et dans un endroit différent à un autre moment ». Certains verbes de mouvement poursuit - il, comme ceux qui nous intéressent ici, et notamment venir, désignent un mouvement délimté. Ils supposent un lieu $P_{1}$, point de départ où le déplacement commence au moment $T_{1}$, et un lieu $P_{2}$, point final où il prend son terme au moment $T_{2}$. Ces paramètres relatifs au référent, c'est-à-dire à l'événement auquel renvoie l'énoncé, peuvent apparaître explicitement sous forme de circonstants :

${ }^{6}$ Voir Langacker R., 1990, p. 236.

${ }^{7}$ Fillmore Ch. J., 1975, p. $51:$ «We say of something that it has moved, in the «locomotion» sense of movement that $I$ have in mind, if it is at one location at one time and at another location at another time ». Cette définition est tout à fait satisfaisante si, comme Fillmore, on s'intéresse au mouvement délimité, en laissant đe côté, par exemple les rotations des corps terrestres et célestes. 
(16) Il est clair que depuis qu'il est arrivé de Metz $\left(P_{1}\right)$ pour environ 3M€ en juin $2000\left(T_{2}\right)$, ses statistiques laissent rêveur (écrit électronique).

(17) Il est arrivé à la gare de Tokyo $\left(P_{2}\right)$ avec trois minutes de retard (écrit électronique).

(18) Il était $10 \mathrm{ho0}\left(T_{1}\right)$ quand nous sommes partis voir une cascade dans les bois et la grotte Notre Dame de Lourdes (écrit électronique).

Ces énoncés renvoient à un déplacement extra-linguistique. Dans le même texte, Fillmore remarque que l'acte d'énonciation peut, lui aussi, être considéré comme un déplacement, un transfert de message entre des interlocuteurs. Liés à la situation d'énonciation, les paramètres de ce transfert sont alors identifiables à l'intérieur de celle-ci. Le message est codé par le locuteur en un lieu (que nous allons noter $P_{m l}$ pour marquer la différence d'avec le $P_{1}$ de référence), à un moment $T_{m 1}$ qui caractérisent ensemble «le départ » du message. Celui-ci est ensuite décodé par son destinataire en un lieu $P_{\mathrm{m} 2}$ au moment $T_{\mathrm{m} 2}$. Il arrive souvent, surtout dans un échange oral qui se déroule en tête à tête, que le codage et le décodage se suivent immédiatement de telle façon qu'il suffit de parler du temps de l'énonciation (du message) $T_{\mathrm{m}}$ en seul et même lieu $P_{\mathrm{m}}$. Or, il existe des verbes qui codent différentes configurations de paramètres référentiels et déictiques à la fois sans recours aux circonstants. C'est le cas de venir qui nous intéresse ici en tant qu'argument de $R E$-.

Imaginons un café avec son lot d'habitués dont l'un a pendant un moment manqué à l'appel. Ses comparses pourront dire avec un brin de reproche bien compréhensible :

(19) Il n'est pas venu depuis un bon moment.

Ajouter un complément « au café » serait redondant. Venir n'en a pas besoin pour orienter l'interprétation de (19) vers le lieu où se termine le déplacement, c'est-à-dire vers le paramètre $P_{2}$ du référent. Le locuteur et le destinataire peuvent se trouver à bonne distance l'un de l'autre $\left(P_{\mathrm{m} 1} \neq P_{\mathrm{m} 2}\right)$ et, s'il est écrit, le message pourra être lu à un moment $T_{\mathrm{m} 2}$ distinct de $T_{\mathrm{m} 1}\left(T_{\mathrm{m} 1} \neq T_{\mathrm{m} 2}\right)$. Dans ce cas précis, la situation d'énonciation, avec son $P_{\mathrm{m}}$ et son $T_{\mathrm{m}}$, importe peu, du moment que $P_{2}$ est présent dans l'univers du discours. L'exemple (20) montre néanmoins que venir exprime quelquefois des configurations de paramètres relatifs à l'acte d'énonciation.

(20) Salut les vieux... je viens à Grenoble du 12/05 au 15/05, y'a moyen de s'organiser un pot ?????? (forum électronique)

Dans cet emploi, venir est en effet suivi d'un circonstant, mais en même temps, il véhicule l'information capitale pour l'interprétation de (20): Grenoble, c'est $P_{\mathrm{m} 2}$. Venir localise ainsi les destinataires du message et le lieu où celui-ci sera décodé. Cette information disparaîtra, si nous remplaçons venir par un autre verbe :

(21) Salut les vieux... je vais à Grenoble du 12/05 au 15/05, y'a moyen de s'organiset un pot ????? 
L'information concernant le déplacement, c'est-à-dire l'événement auquel réfère (21), n'est pas différente de (20), mais Grenoble n'y a pas le statut de $P_{\mathrm{m} 2}{ }^{8}$

2.1.2.2. Revenons (si je puis dire) à l'exemple du café évoqué plus haut, qui constituera de nouveau notre univers du discours. Le compagnon perdu de vue réapparaît, fait auquel on pourra référer par (22):

(22) Il est enfin revenu.

$R E$ - avec sa valeur «à nouveau» porte ici sur la totalité du schéma actanciel « $A$ vient à $P_{2}$ » constitué par l'agent et la variable $P_{2}$ identifiable à l'intérieur de l'univers du discours. En d'autres termes, l'énoncé (22) exprime une itération du procès. Nous obtiendrons un résultat comparable en appliquant $R E$ - au venir de l'exemple (20):

(23) Salut les vieux... je reviens à Grenoble du 12/05 au 15/05, y'a moyen de s'organiser un pot ??????

à une différence près. Dans l'interprétation de (23), à la place de l'argument $P$, nous trouverons le paramètre déictique $P_{\mathrm{m} 2}$, relatif à l'emplacement du destinataire du message au moment du décodage. L'information présupposée valable dans l'univers du discours peut être formulée de manière suivante : " $A$ est déjà venu à Grenoble ». Nous admettons cependant volontiers que, pris en dehors de tout contexte (au sens large du terme), et cela malgré la spécification temporclle du circonstant, (23) est ambigu. A côté de l'interprétation itérative, il peut prêter à une glose annulative : «A vient à nouveau pour être dans un lieu (Grenoble) qu'il avait auparavant quitté » et l'information présupposée valide dans l'univers du discours sera alors " $A$ avait quitté $P$ ». C'est aussi dans cette interprétation itérative, qu'il faut prendre l'exemple (5) dont nous parlerons ultérieurement.

Le foncteur $R E$ - adjoint à venir conduit, comme nous venons de le voir, à des effets de sens itératifs et annulatifs. Il semblerait cependant que seuls deux des trois types d'itérativité apparaissent sous son action. Le cas de l'itération du procès nous paraît attesté par (22) et (23). L'itération de l'activité nous semble également possible : c'cst cet effet de sens que l'on pourrait accorder aux exemples (13) et (14) ci-dessus. On peut les paraphraser de manière suivante «à nouveau $A$ vient ». Le foncteur $R E$ - porte seulement sur « $A$ vient $»$, et non pas sur $P^{9}$.

2.1.3. Pour clore provisoirement ces réflexions autour du verbe revenir, il convient de remarquer qu'il satisfait également à la troisième condition évoquée à

${ }^{8}$ Pour éviter de compliquer notre exposé, nous laisssons (ici) de côté le jeu de ces paramètres autour du personnage considéré comme central dans les textes narratifs.

${ }^{9}$ Dans la mesure où il s'agit de communiqués à caractère publicitaire, on peut se demander si le destinataire du message n'est pas à chaque fois celui ou celle qui le lit (en $P_{\mathrm{m} 2}$ au moment $T_{\mathrm{m} 2}$ ) auquel cas, le paramètre $P_{\mathrm{m} 2}$ se trouverait également dans la champ du foncteur et nous serions face à une itération du procès. 
la fin de la section 1. Tant dans ses acceptions itératives, que cette itérativité concerne le procès ou l'activité, que dans son interprétation annulative, revenir est bel et bien un hyponyme de sa base de dérivation, c'est-à-dire de venir. Les gloses que nous avons proposées montrent que grâce à l'apport sémantique de $R E$-, revenir véhicule une spécification supplémentaire par rapport à son hyperonyme. Nous pouvons donc le compter parmi les mots construits du lexique résultant du schème dérivationnel en $R E$ - et, par conséquent l'analyser en ces termes.

\subsection{Le cas de rentrer ${ }^{10}$}

Devant l'initiale vocalique nasale de entrer, $R E$ - apparaît sous sa forme $r$-, l'un de ses allomorphes. Ce n'est d'ailleurs pas sa seule variante auprès de entrer, puisqu'on note aussi des formes en ré- et en re-

2.2.1. Le cas de rentrer confirme l'observation d'Apothéloz (2005), selon laquelle une spécialisation sémantique accompagne les variations allomorphique du foncteur $R E$-. Ainsi, rentrer relève d'un microsystème d'opposition lexicale composé de couples de dérivés avec une même base. C'est notamment le cas de raccompagner vs reaccompagner, de racheter vs reacheter, etc. Les premiers membres de ces paires véhiculent surtout une valeur annulative et éventuellement itérative, alors que leurs doublons en re- expriment systématiquement une itération ${ }^{11}$. Nos observations concernant entrer et ses dérivés en $R E$ - vont dans le même sens. L'exemple (24) nous semble éloquent à ce titre :

(24) Les bureaux de direction? Il faut ressortir d'ici et rentrer par la porte principale.

L'interprétation que I'on donnera à (24) ne va pas de soi. On peut la concevoir comme un effet de sens itératif avec une paraphrase telle que : «il faut que $A$ entre à nouveau dans $P$ ». Cette interprétation ne sera cependant acceptable qu'à une seule condition : on doit la considérer comme une itération du procès. Entrer et rentrer doivent avoir le même paramètre $P$. Dans le cas (24), ce sera bien évidemment non pas la porte, mais le bâtiment, c'est-à-dire l'entité dont la ou les portes incriminée(s) font partie. En effet, (25) paraît difficilement, voire pas du tout acceptable :

? (25) Les bureaux de direction? Il faut ressortir et rentrer dans le bâtiment derrière.

La contrainte d'un seul et même $P$, qui pèse sur (24) est à la base de l'ambiguïté que présente cet énoncé. A côté de l'interprétation itérative que nous venons de proposer, une lecture annulative est également possible : " $A$ doit entrer pour être à nouveau dans $P_{\text {}}$. Pour lever l'ambiguïté, le locuteur peut recourir à $r \dot{~}^{\prime 2}$, allomorphe du préfixe $R E-$, à valeur itérative :

${ }^{10}$ Dans le cadre de cet article, seuls les emplois intransitifs de rentrer et de entrer nous intéressent. Aussi n'allons nous pas nous arrêter sur les emplois tels que renter sa voiture.

"Voir A pothéloz (2005), p. 64-67.

${ }^{12}$ Sans prétendre ériger cctte observation au rang de système, il semblerait que l'allomorphe re- à valcur iterative soit plus fréquent quand il s'agit de entrer dans les emplois intransitifs : "Si vous 
(26) Une fois dans le parc, peut-on ressortir et ré-entrer?

Oui, vous pouvez quitter le parc, un tampon sera mis sur votre main pour pouvoir ré-entrer dans la même journée. (site électronique d'un parc Walibi)

D'ores et déjà, nous pouvons constater que les conditions (i) et (iii) sont satisfaites. Rentrer et ré-entrer sont des mots construits appartenant au même paradigme dérivationnel en $R E$-. Ils produisent bien des effets de sens itératif et annulatif, qui à un niveau d'analyse ou un autre comprennent l'élément «à nouveau» (i). Par ailleurs, leurs sens apporte des spécifications supplémentaires par rapport à celui de leur base de dérivation, ce qui nous permet de conclure à une relation d'hyponymie que rentrer et ré-entrer entretiennent avec entrer (iii). Avant de vérifier si la condition (ii) est également remplie, il nous paraît intéressant de s'arrêter sur la question des types d'itérativité véhiculés par rentrer.

Dans le cas de rentrer intransitif, comme d'ailleurs dans celui de revenir, la structure actancielle met en jeu les mêmes paramètres. Dans les exemples que nous avons vus jusqu'ici, l'agent du déplacement est en même temps le patient ou le bénéficiaire, c'est-à-dire respectivement celui qui le subit ou qui en profite. Il parâ̂t donc difficilement imaginable de séparer les deux. Logiquement, le foncteur $R E$ - ne saurait porter uniquement sur le patient, sans opérer sur l'agent. Une itération de l'événement, comme dans l'exemple (9), semble impossible en ce qui concerne revenir et rentrer. Et pourtant certains emplois de ce dernier conduisent bel et bien à cet effet de sens. L'exemple (27) montre bien de quoi il s'agit, si nous l'envisageons à travers une situation d'énonciation et un univers du discours, c'est-à-dire à travers des caractéristiques pragmatiques qui accompagnent le message. La première pourrait, pour les besoins de notre démonstration, se résumer de cette façon : $A$ et $B$ sont nos interlocuteurs, tour à tour auteur et destinataire des énoncés qui composent l'échange (27). Pour compléter l'image, précisons que $A$ sonne pour la première fois de sa vie à la porte de $B$. Comme on l'a entendu maintes fois, $B$ peut alors dire en ouvrant :

(27) $B:$ Rentre !

$A$ : Une autre fois volontiers. Là, je n'ai pas le temps d'entrer.

De toute évidence, $A$ et $B$ réfèrent à une même situation extra-linguistique. Les items différents qu'ils emploient à cette fin ne peuvent s'expliquer qu'à l'interieur de leurs univers du discours respectifs. Dans la cas de $A$, il s'agit bien d'une première réalisation de l'activité consistant à entrer ${ }^{13}$. Dans son univers du discours, $B$ n'a pas la même conceptualisation des choses. Compte tenu du paramètre $P_{\mathrm{m}} \mathrm{du}$ message, c'est-à-dire de l'endroit où il l'émet, il envisage l'action consistant à entrer dans son appartement comme un événement reproduit phus ou moins souvent

supprimez vos cookies, vous devez re-entrer tous vos mots de passe de connexion et de messagerie " (écrit électronique).

${ }^{13} \mathrm{~S}^{\prime}$ il compte la reproduire dans certaines conditions, il pourra toujours recourir à ré-. 
par des agents différents. L'invitation à entrer dans (27) accepte, sans rien enlever à l'hospitalié de $A$, la paraphrase : «à nouveau il y a une entrée ».

2.2.2. Suivant la condition (ii), le foncteur $R E$ - opère sur un ou plusieurs paramètres de la structure sémantique de entrer. Sa portée détermine ensuite l'une des trois interprétations itératives ou bien annulative à donner. Nous avons vu, dans la section 2.1, que les paramètres discursifs de venir sont également présents dans revenir. Si un prédicat comme aimer établit une relation entre un agent et un patient, les verbes de déplacement construisent un rapport entre "cible l'entité localisée et site le point de repère par rapport auquel sa situation est fixée $»^{14}$. Il nous semble que le sens du terme site employé par Vandeloise n'est pas sensiblement différent de ce que nous désignons par $P$. S'il ne permet pas de faire la différence entre les types de $P$ distingués par Fillmore, sa définition n'en saisit pas moins la nature du rapport entre ces paramètres des verbes de déplacement. Le verbe luimême impose certaines contraintes quant à $P$. Aussi venir comme revenir, orientent-ils la cible vers $P_{2}$, que ce soit $P_{2}$ ou $P_{\mathrm{tr} 2}$, la seule façon pour eux de référer à $P_{1}$ étant de recourir, sur le plan syntaxique, à un complément de lieu. Entrer, quant à lui, tout en se focalisant sur $P_{2}$, fait peser des contraintes supplémentaires :

(28) Ce matin, je suis entré dans la bibliothèque-fouillis dont j'ai l'babitude (écrit électronique).

(29) Je suis entré au club de Valenciennes comme responsable administratif (écrit électronique).

(30) Depuis le jour oú je suis entré sur le marché du travail, je n'ai accompli que des boulots vains, sans intérêt réel (écrit électronique).

Certaines prépositions, par exemple vers n'introduisent quasiment jamais le complément de lieu de entrer. C'est parce que celui-ci, par son sémantisme, conceptualise $P$ en tant qu'entité bi ou tridimensionnelle délimitée dans l'espace, de manière à ce que l'agent puisse, au terme du procès, se retrouver à l'intérieur de celle-ci. En d'autres termes, entrer impose une relation contenu-contenant entre l'agent (ou la cible, si l'on préfère) et $P$, le site, qu'il soit référentiel ou pragmatique ${ }^{15}$. On pourrait, certes, objecter que du point de vue matériel, (30) contrevient à ce que nous venons de dire : le marché du travail n'est pas - heureusement pour les demandeurs d'emploi - spatialement délimité. Nous répondtons alors, en suivant l'étude de Vandeloise (1987) consacrée à la préposition $\grave{a}$, que ce rapport locatif peut s'étendre, par métaphore, à des sites institutionnels et à la routine qui leur est associée. Ils sont alors conceptualisés comme entités capables de contenir ou d'inclure, indépendamment de leur caractère spatial ou non dans la réalité. La contrainte reste valide lorsque le paramètre $P$ tombe dans la portée de $R E$-. Nous tenons donc la réponse à la question de savoir pourquoi, l'exemple (6) est inacceptable dans la

\footnotetext{
${ }^{14}$ Vandeloise C. (1987), p. 77.

${ }^{15}$ Borillo qualifie cette relation de rapport d'inclusion (voir Borillo, 1998).
} 
situation d'énonciation décrite dans la section (1). Si, dans l'exemple (5), on ne peut pas subsutituer tout bonnement rentrer à revenir :

(5) Va t'acheter une glace et reviens.

? (6) Va t'acheter une glace et rentre.

Dans cet emploi, revenir s'interprète de façon annulative : "viens pour être à nouveau à l'endroit que tu quittes ". Le site auquel réfere le locuteur-auteur de (5) est celui où il se trouve au moment de prononcer le message décodé aussitôt par le destinataire au moment $T_{\mathrm{m}}$. $R E$ - porte donc sur le paramètre $P_{\mathrm{m}}$ de venir qui est, certes, un point de référence spatiale, mais qui n'est pas conceptualisé comme contenant. Revenir et rentrer redeviennent substituables dès qu'une telle conceptualisation est possible. Nous supprimerions sans hésiter le point d'interrogation de (6), si $P$ désignait une maison, un appartement ou ne serait-ce qu'une tente.

\subsection{Le cas de retourner ${ }^{16}$}

2.3.1. Malgré un air de famille morphologique, retourner, contrairement à revenir et à rentrer, ne peut pas (plus ?), dans la synchronie actuelle, être compté parmi les mots construits en $R E$-. Certes, l'élément « $\dot{a}$ nouveau» est facile à y trouver, comme le montrent les exemples (31) et (32):

(31) Je suis revenu de Minsk paris/varsovie en avion ensuite varsovie/minsk en train aucun problème dans mon hôtel je suis resté 2 semaines je pense $y$ retourner bientôt (frederic, Paris, écrit électronique, ortographe de l'original)

(32) Crespo retourne à Chelsea (dépêche AFP)

Dans l'exemple (31), retourner produit un effet de sens itératif que l'on peut gloser par « $y$ aller à nouveau ». Le schème actanciel est reproduit dans sa totalité, ce qui permet de conclure à une itération du procès. Dans (32), nous avons une valeur annulative paraphrasable par : «A retourne à l'endroit qu'il avait auparavant quitté ». Le contexte situationnel de l'énoncé confirme cette interprétation puisque la même dépêche de l'Agence France-Presse précise que le footballeur-vedette avait été prêté pendant un an au Milan AC.

2.3.2. La présence du composant "à nouveau " ne suffit pas pour compter retourner parmi les construits en $R E$-, alors que les conditions (ii) et (iii) ne sont pas

${ }^{16}$ Dans le cadre de cet article, seuls les emplois intransitifs de retourner paraphrasables par aller à nous intéressent. La place nous manque pour en faire un exposé complet, mais disons, en résumé, que retourner remplit les trois conditions, et donc est bien un mot construit dans les exemples suivants : (a) "Il se retournait dans tous les sens sans trouver le sommeil " ou (b) "Ce n'est pas la première fois qu'elle a retourné sa veste. » Le foncteur opère alors sur la base (se) tourner en produisant des effets de sens intćratifs (a) ou annulatifs (b). 
remplies. Les valeurs itérative et annulative de (31) et de (32) ne sauraient être considérées, dans une perspective synchronique actuelle, comme issues de $R E$ - opérant sur la base tourner, ni sur aucun de ses paramètres. Ils ne sont pas non plus hyponymes de tourner, ni lorsque celui-ci désigne un mouvement rotatif, ni quand il s'agit d'un changement de direction. Si relation d'hyponymie il y a, c'est plutôt par rapport à aller.

Les conditions (ii) et (iii) n'étant pas remplies, nous ne pouvons pas (plus?) voir en retourner un construit en $R E$-. S'il l'a été, à un moment ou un autre de sa diachronie, il a perdu sa compositionnalité et ne peut plus être analysé en termes des apports sémantiques relatifs au préfixe et à la base de dérivation. Il a subi un processus de lexicalisation en codant dans son signifié ce que nous interprétons aujourd'hui comme effet de sens itératif et annulatif.

2.3.3. Cependant, ce constat ne permet pas d'expliquer pourquoi, malgré les effets de sens apparemment comparables, revenir et retourner ne sont pas subsitituables, par exemple dans (5) et (7). En cherchant la réponse, observons d'abord une régularité syntaxique, qui à notre avis, constitue un indice important. Or, retourner, contrairement à revenir et rentrer, appelle systématiquement un complément de lieu soit sous forme d'un syntagme nominal, soit sous une forme pronominale ou adjectivale indexicale (ici dans l'exemple 7). Ce complément, présent au niveau syntaxique, est un complément directionnel indiquant $P_{2}$, c'est-à-dire le lieu où le mouvement auquel réfëre retourner prend sa fin. Or, dans les cas de revenir et de rentrer une telle complémentation apparaît quelquefois sur le plan syntaxique, mais elle n'est pas systématique. Rien d'étonnant à cela, puisque, rappelons-le, ces verbes orientent l'interprétation vers un $P_{2}$ identifié dans l'univers du discours et/ou vers un $P_{\mathrm{m} 2}$ lié au locuteur au moment d'énonciation (exemple 5) ou bien au locuteur au moment où le message est supposé lui parvenir (exemple 23). Si retourner appelle systématiquement un complément directionnel, c'est que, de par son sémantisme, il ne renvoie pas automatiquement au paramètre $\mathrm{P}_{2}$ quel qu'il soit. Notre hypothèse est que, au contraire, le paramètre central de retourner, c'est $P_{1}$. L'exemple (33) va tout à fait dans ce sens :

(33) Discovery retourne dans l'espace. (titre de presse)

puisqu'il est possible de le paraphraser intuitivement par : «A quitte $P_{1}$ pour aller à nouveau a/dans/sur $P_{2} \gg$. Dans ce cas, il semblerait que les deux paramètres $P_{1}$ entrent en jeu: le $P_{1}$ du référent, c'est-à-dire le lieu initial où le déplacement commence, et le $P_{\mathrm{ml}}$ relatif à la position de l'auteur de l'énoncé. C'est d'ailleurs ce demier qui se revèle décisif. Remplaçons, dans l'exemple (33), le verbe retourner par revenir. Nous avons alors l'énoncé (34):

(34) Discovery revient dans l'espace. 
qui est difficilement acceptable, sauf ... s'il est énoncé par un membre de l'équipe à bord de la Station internationale qui signifiera ainsi " $A$ vient à nouveau à/dans/sur $P_{\mathrm{nL} 2}$ ", c'est-à-dire à l'endroit où se trouve le locuteur.

Si avec revenir et rentrer, nous accordons un statut privilégié à $P_{2}$, l'entrée en jeu de $P_{1}$ introduit un élément nouveau dans les interprétations de retourner. Puisque nous faisons référence, dans un même énoncé à $P_{1}$ et à $P_{2}$, nous ne pouvons ignorer la distance qui les sépare, même si, dans la plupart, elle n'est pas des cas précisée. L'état résultatif prévu du procès se laisse résumer de manière suivante : «A n'est plus à $P_{1}, A$ est à $P_{2}$ ». Pour revenir et retourner, on dira seulement : «A est à $P_{2} »$.

2.3.4. Un dernier point mérite, à notre avis, l'attention, puisqu'il s'avère décisif pour la distribution de retourner. Dans la section 2.2.1, noue avons constaté, en ce qui concerne revenir et rentrer ${ }^{17}$, que l'agent du déplacement en est en même temps patient ou bénéficaire. En d'autres termes, le sujet grammatical renvoie à l'agent. Ce n'est pas toujours le cas pour retourner:

(35) Tu peux prendre mon dictionnaire, il est sur l'étagère, mais après, il doit retourner à sa place.

Le sujet grammatical de (35) renvoie non pas à l'agent du déplacement mais à son bénéficiaire. Nous tenons ainsi un autre élément de réponse à la question pourquoi la substitution pure et simple des trois verbes n'est pas toujours possible.

\section{LE CAS DE WRÓCIĆ/WRACAĆ}

Ce couple aspectuel est sémantiquement proche des verbes français que nous venons d'analyser. $\mathrm{Si}$, dans une approche synchronique actuelle, on analyse ces deux verbes d'un point de vue constructionnel, ce n'est pas en tant que mots construits, mais uniquement dans la mesure où ils servent de base de dérivation par préfixation en po- et od-. En d'autres termes, wrócić et wracać sont des items lexicalisés, au sens où ils codent un signifié qui n'est pas calculable à partir de leur structure morphologique. Ils appartiennent au lexique mental des usagers de la langue qui y accèdent par voie mémorielle.

\subsection{Une itération est-elle possible?}

Les énoncés dont l'un des deux est le prédicat rendent compte du même type d'événément comprenant un déplacement. Ils partagent la plupart des valeurs de revenir, rentrer et retourner, sans pour autant être leurs miroirs fidèles. Pour un francophone, le maniement de wracać/wrócić, qui pourrait paraitre plus simple pour

${ }^{17}$ Nous pouvons, certes, dire que la voiture rentre dans le garage, mais nous allons alors référer à un rapport locatif dimensionnel et non pas à un déplacement. 
des raisons quantitatives (les valeurs relatives à trois unités sont à répartir entre deux items), présente cependant quelques embûches. (36) a été énoncé par un francophone parlant polonais. Ajoutons que le locuteur est un provincial effectuant des séjours occasionnels dans la capitale.

(36) Niedlugo wrócimy do Paryża.

Si l'on ne peut rien reprocher à sa grammaticalité, il n'en est pas pour autant plus adéquat à la situation. Compte tenu de $P_{1}$, point de référence spatiale relatif au locuteur, c'est-à-dire au lieu initial de ce déplacement, différent de $P_{2}$, son terme, (36) devait être la traduction de :

(37) Nous allons bientôt retourner à Paris.

dans son acception itérative, effet de sens qui n'est pas celui que produisent wracać ou wrócić. Le procès susceptible d'être reproduit se présente sous forme d'un schéma simple (fig. 1):

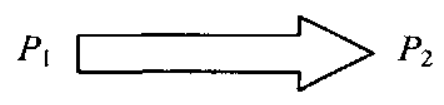

Or, pour exprimer une itération du procès, le polonais recourt à une forme adverbiale adjointe au verbe :

(37') Niedługo znów pojedziemy do Paryża.

Il existe cependant des emplois de wracać/wrócić que l'on pourrait très bien interpréter comme itératifs. C'est le cas de (38) et (39) :

(38) Raz przyjedziesz - wrócisz na pewno.

(Une fois venu, tu reviendras à coup sûr - slogan publicitaire sur le site internet de la ville de Dźwirzyno, petite station balnéaire au bord de la Baltique).

(39) Atmosfcra w mieścic już się uspokoiła, trwa sprzątanie i myślę, że turyści mogą tu już do nas wracać. (Dans la ville. l'atmosphère s'est rasséréneee, on est en train de nettoyer et je pense que les touristes peuvent déjà revenir ici, chez nous - journal télévisé, relation en direct de Sharm el Sheikh par la représentante d'une agence de voyages résidant sur place, peu après les attentats d'août 2005).

Une interprétation itérative ne saurait être exclue à la légère. On dirait alors que wrócić (38) et wracać (39) signifient ici przyjechać/przyjeżdżać ponownie. Nous pourrions ainsi ramener ces énoncés à des formes plus simples, exprimées en français par "venir à nouveau ». Il nous semble cependant que, une fois de plus, il faut prendre en compte les éléments contextuels tels que le statut du locuteur, la convention sociale liée au messages publicitaires, les habitudes culturelles (séjours de vacances). Dans ce contexte, une interprétation annulative n'est pas moins justifiée. Elle s'exprime par la paraphrase "venir pour être à nouveau à $P_{2}$ ». S'agit-il 
de "venir à nouveau » ou plutôt de son état résultatif «être à nouveau à $P_{2}$ "? La seconde interprétation nous semble avoir tout pour elle, ce qui nous fait pencher pour un effet de sens annulatif. Nous reconnaissons néanmoins une certaine ambiguïté due, à notre avis, au rapport entre cause et effet qui relie les deux procès.

\subsection{Wrócić/wracać et la valeur annulative}

Les effets de sens produits par wracać et wrócić sont donc, malgré quelques cas douteux, à classer comme annulatifs. C'est cette valeur que le couple lexicalise et c'est cette interprétation qu'un destinataire polonophone donnera à (36). Dans l'univers du discours, il présuppose valide non pas l'information selon laquelle (a) " $A$ est déjà allé auparavant de $P_{1}$ à $P_{2}$ ", mais celle que l'on peut intuitivement énoncer sous la forme (b): " $A$ avait auparavant quitté $P_{2}$ pour un $P_{1}$ 》. Il active ainsi un tout autre schéma (fig. 2) :

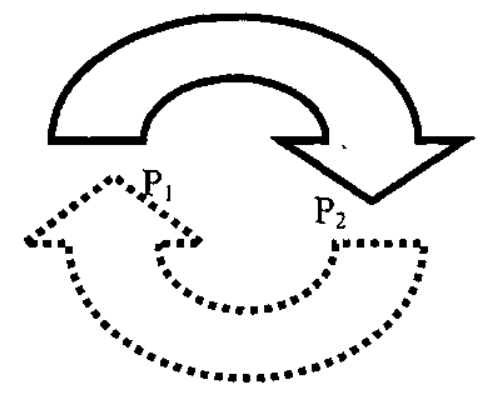

Le mouvement de $P_{2}$ vers $P_{1}$ que représente la flèche en pointillés, correspond à l'information présupposée (b). Il s'agit du procès transformateur qui, dans l'univers du discours, a précédé l'événement auquel réfère le verbe annulatif, en l'occurrence wracać/wrócić, représenté dans notre schéma (fig.2) par la flèche du haut. Ils ne peuvent s'interpréter que par rapport à leur antonymes discursifs tels que odejść, wyjechać, wyjść (équivalents de partir, sortir, quitter,...). Ces derniers, relatifs à un déplacement en direction opposée, n'ont pas besoin d'être énoncés; il suffit qu'ils soient présupposés.

Wrócić/wracać privilégient le $P$ relatif au terme du déplacement. Cependant, comme le laisse voir le schéma ci-dessus, ce $P$ final présente une particularité : en tant que point de référence spatiale, il est en même temps le lieu initial du procès exprimé par l'antonyme discursif, celui-là même qui se rapporte au procès transformateur présupposé. C'est ainsi que nous allons interpréter l'exemple (4) de la section 1. C'est également en fonction de ce statut privilégié du $P$ final, que (40) ne peut être compris que dans un sens annulatif.

(38) Po chwili wrócila. Pomyślałem, że musi mieszkać bardzo blisko.

Le $P$ final du déplacement est identifié par rapport à la position du locuteur au moment de référence et éventuellement au moment d'énonciation. Puisque nous ne 
disposons d'aucune information concernant les caractéristiques dimensionnelles de $P$, pour la traduction, il nous faut recourir à revenir :

(40') Elle est revenue au bout d'un moment. Je me suis dit qu'elle devait habiter tout près.

\subsection{Wrócić et wracać versus retourner}

3.3.1. Les propriétés actancielles de wrócić et wracać d'un côté, de retourner de l'autre, présentent une certaine ressemblance. Dans le rôle de leur sujet grammatical, nous trouvons aussi bien l'agent du déplacement, comme dans les exemples (40) et (41) que son bénéficiaire comme dans l'exemple (42).

(41) Tym razem wrócę z wakacji opalona i wspaniale wypoczęta (Cette fois, je reviendrai de vacances bronzée et merveilleusement reposée - pressc).

(42) Codex Iuris Lubschicensis powinien wrócić do Głubczyc (= Le CIL devrait retourner à Gtubczyce $\cdots$ presse).

\subsubsection{Une conceptualisation différente}

Un énoncé avec wrócić ou wracać dans le rôle du prédicat peut spécifier $P_{1}$ par un complément de lieu, comme cela a lieu dans (41), mais les deux verbes restent orientés vers $P_{2}$. C'est ce paramètre qu'ils ont en commun avec revenir et rentrer, même si, contrairement à ce dernier, en polonais aucune contrainte n'est imposée quant aux caractéristiques dimensionnelles de $P_{2}$. Qu'en est-il donc de $P_{1}$ dans le couple wrócić/wracać? Encore une fois, des éléments contextuels qui accompagnent la situation d'énonciation seront d'une grande utilité. L'exemple qui suit a été puisé dans la vie quotidienne. Sur la porte fermée d'une boutique, il n'est pas rare de tomber sur l'écriteau dans le genre de (43) laissé par employé qui a dû s'absenter :

(43) Zaraz wracam.

Laissons de côté les références temporelles concernant le message pour n'envisager que les paramètres spatiaux. Dans (43), wracać fait référence à $P_{2}$, qui est à la fois le terme du déplacement de l'agent-locuteur et le lieu où son message parviendra à l'allocutaire. Dans une situation similaire en France, nous avons trouvé (44) sur la porte d'une boutique de photocopies.

(44) Il est 10 heures 30 . Je reviens dans 20 minutes.

Quelle que soit la raison de cette défection, le locuteur-agent se trouve à un endroit $P_{1}$, différent de $P_{2}$, où son mouvement annulatif commencera. Au moment de se mettre en route pour rejoindre son lieu de travail, il dit en polonais :

(45) No dobra, muszę wraca/wrócić do sklepu résolution louable que l'on exprimera en français par :

(46) Bon, il faut que je retourne au magasin.

Le verbe polonais wracać et son doublon perfectif wrócić rendent compte tous les deux des situations envisagées dans (44) et (46) pour lesquelles le français dispose de deux verbes différents. Il oriente l'activité vers son pôle final $P_{2}$ sans subir les 
contraintes qui, en français, obligent le locuteur à choisir entre revenir et rentrer, ce dernier étant marqué par les caractéristiques dimensionnelles et le rapport d'inclusion imposé à $P_{2}$. Une autre différence réside dans la conceptualisation des paramètres situationnels en jeu lorsqu'il s'agit de retourner. Contrairement au polonais, le français prend en compte la position initiale de $A$ et/ou celle du locuteur par rapport au déroulement du procès. Peut-on pour autant parler d'une polarité initiale pour retourner? Il nous semble que non. Retourner, comme ses «proches » sémantiques français et polonais à l'exception de wracać qui désigne une activité, est un verbe télique supposant, certes, une durée, mais porté essentiellement vers le résultat qui sera son aboutissement. D'un point de vue cognitif, un procès de déplacement suppose un scénario. En transposant cette idée de scénario dans un domaine théâtral, on pourra dire que les éléments de la scène (actancielle) prévus dans le script, se trouvent mis au premicr plan grâce à un jeu de lumières savamment orchestré, alors que d'autres restent dans l'ombre. Dans le cas de wrócić/wracać, comme dans celui de revenir et rentrer, c'est $P_{2}$ qui est mis en avant. Avec retourner il n'est nullement perdu de vue mais on le regarde, en tant que $A$ ou en tant que locuteur, par rapport à $P_{1}$. La "gestion" de tous ces paramètres et le choix lexical qu'elle soutend se compliquent inévitablement quand le nombre de protagonistes augmente dans un texte ou dans un discours. La dépêche de l'Agence France-Presse dont nous avons donné le titre dans l'exemple (32), continue en introduisant des points de référence spatiale liés aussi bien à son auteur qu'aux deux protagonistes : Hernan Crespo et Peter Kenyon :

(32) Crespo retourne à Chelsea

(32') "Nous sommes ravis que Hernan revienne " a déclaré le directeur général du club londonien Peter Kenyon sur www. Chelseafc.com. «Il a réalisé une grande saison pendant son prêt à Milan (...) et il est heureux de revenir à Stamford Bridge... "

Le recours à retourner dans le titre, puis à revenir lorsqu'un autre protagoniste intervient, permet ainsi de localiser avec plus ou moins de précision tous les intervenants. En ce qui concerne le locuteur, cette localisation est négative : il ne se situe pas à Londres, et encore moins à Chelsea. Hernan Crespo, au moment d'énonciation, se trouve encore à Milan, et Peter Kenyon est bien chez lui, lieu qui signifie le terme du mouvement $P_{2}$. Bien évidemment, il ne s'agit pas de la localisation réelle des protagonistes au moment du discours mais à leur positionnement par le locuteur par rapport au procès. L'analyse est simpliste, voire grossière, mais son unique but est de montrer la complexité de ce jeu des paramètres spatiaux que l'on doit affronter lors du processus d'apprentissage du français langue étrangère.

\section{POUR RÉCAPITULER}

Selon les langues, nous parlons d'un même événement ou état des choses sans forcément mettre en avant les mêmes paramètres. Pour un Polonais, choisir entre revenir ou retourner suppose de se situer par rapport à un paramètre $P_{1}$ qui, pour le 
type de procès décrit, n'a pas la même saillance cognitive dans sa langue maternelle. Le risque est alors d'aboutir à un hiatus entre ce que Donnellan (1978) appelle la référence du locuteur (what the speaker had in mind) et la référence sémantique, c'est-à-dire le sens qu'a conventionnellement une expression dans une langue. Le sens descriptif conventionnel est sans doute plus facile à saisir dans une situation d'apprentissage. Or, pour manier correctement les verbes dont nous venons de parler, il faut mâ̂triser en plus leur sens instructionnel, c'est-à-dire celui qui indique les procédures indispensables pour identifier le référent.

Pour terminer, essayons de réunir dans un tableau les caractéristiques sémantiques et pragmatiques des verbes qui nous ont occupés jusque là.

\begin{tabular}{|c|c|c|c|c|}
\hline verbe & wracać/wrócić & revenir & rentrer & retourner \\
\hline $\begin{array}{c}\text { valeur } \\
\text { valeur(s) } \\
\text { térative(s) }\end{array}$ & - & + & + & + \\
\hline valeur annulative & + & + & + & + \\
\hline $\begin{array}{c}\text { point de } \\
\text { référence spatiale } \\
\text { visé }\end{array}$ & $P_{2}$ & $P_{2}$ & $P_{2}$ & $\begin{array}{c}P_{2} \text { envisagé } \\
\text { depuis } P_{1}\end{array}$ \\
\hline $\begin{array}{c}\text { contraintes } \\
\text { sćmantiques sur } \\
P_{2}\end{array}$ & - & - & $\begin{array}{c}\text { rapport } \\
\text { d'inclusion entre } \\
P_{2} \text { et } x\end{array}$ & - \\
\hline $\begin{array}{c}\text { cas profond du } \\
\text { sujet } \\
\text { grammatical }\end{array}$ & bénéficaire & agent & agent & bénéficaire \\
\hline
\end{tabular}

Il nous reste à répondre à la question qui ouvre cet article. On revient à ses moutons dans un sens annulatif, vers un $P_{2}$ où il nous faut se trouver à nouveau. On y rentre pas, car le point de référence spatiale ne suppose pas un rapport d'inclusion (il ne s'agit pas de rentrer dans une bergerie). On ne peut pas retoumer à ses moutons pour deux raisons. Premièrement pour cause d'agrammaticalité : à ses moutons, tout en étant un point de référence spatiale, indépendamment de ses emplois métaphoriques, ne saurait être un complément de lieu, le test en où faisant foi :

(47) - Où voulez-vous revenir?

- ? A nos moutons.

Deuxièmement, le procès annulatif est focalisé sur $P_{2}$ final, et non pas sur $P_{1}$ point de départ. 


\section{BIBLIOGRAPHIE}

A pothéloz D. (2005), RE- et les différentes manifestations de l'itérativité, Pratiques, 125-126, p. 4871.

A pothéloz D., Pekarek Doehler S. (2003), Nouvelles perspectives sur la référence : des approches informationnelles aux approches interactionnelles, Verbum XXV, 2, p. 109-136.

Borillo A. (1998), L espace et son expression en français, Ophrys, Paris.

Donnellan K. (1978), Speaker Reference, Descriptions and Anaphora, P. Cole éd., Pragmatics, Syntax and Semantics, 9, 47-68.

Fillmore Ch.J. (1975), Santa Cruz Lectures on Deixis : Coming and Going, Indiana University Linguistics Club.

Kleiber G. (1999), Problèmes de sémantique. La polysémie en questions, Presses Universitaires du Septentrion.

Langacker R.W. (1990), Concept, image and symbol. The cognitive basis of grammar, Mouton de Gruyter, Berlin - New York.

Moesc hler J. (1994), Dictionnaire encyclopédique de pragmatique, Seuil, Paris.

Przybylska R. (2002), Polisemia przyimków polskich w świetle semantyki kognitywnej. (Polysémie des prépositions polonaises dans une perspective sémantique cognitive), Universitas, Kraków.

Slobin D. (2003), Od " myśli i jezzyka » do " myślenia dla mówienia ». Trad. O. Kubińska, W. Kubiński. Dans : E. Dąbrowska, W. Kubiński (réd.), Akwizycja języka w'świetle językoznawstwa kognitywnego. Wydawnictwo Universitas, Kraków, p. 361-402.

Vandelois e C. (1987), La préposition à et le principe d'anticipation, Langue Française, 76, p. $77-$ 111. 